\title{
Neutral citation is poor scholarship
}

\author{
Citation of prior publications is essential both to claim that knowledge is needed in your area of research and to \\ establish that you have indeed advanced understanding substantially in that area. The journal deplores and will \\ decline to consider manuscripts that fail to identify the key findings of published articles and that-deliberately or \\ inadvertently-omit the reason the prior work is cited.
}

0 ur examination of articles we have recently published identifies three flavors of citation. Of these, the most accurate is methodological citation to laboratory and informatics techniques. The rarest by far are mentions of supporting or contradictory publications. Indeed, many publications contain absolutely no published references supporting or refuting a main claim or finding. Weak evidence of consistency from a different approach may be mentioned, but we suspect that strong prior evidence and robust challenges are somewhat underreported relative to the current advance. Neutral, flavorless or unexamined mention predominates, and we believe this to be an increasing problem for the integrity of scientific communication, whether it is used in the Introduction or in the Results and Discussion.

Neutral citation, for example, "this field exists (refs. 1-20)," may on the face of it seem to be a fair practice, giving evenhanded and minimal citation credit to a range of preexisting works as background to the current report. But it can also be malpractice, artificially inflating the metrics of irrelevant or trivially related works by including them in lists of relevant publications. However, the misdeed that most enrages editors, referees and readers alike is misrepresentation of published achievement. If a previous publication introduced a new concept, it needs to be cited in a way that acknowledges the concept accurately. Similarly, if the prior work showed by experiment or analysis that a concept was falsified or supported, then it is inadequate merely to acknowledge that the prior work exists. In this respect, neutral citation can minimize or neutralize the findings of other researchers and misrepresent the current work as the major advance in the field.
Best citation practice is to summarize the claim made in the cited work without distorting whether it was of cause, correlation or conjecture, much as you would for your own findings (Nat. Genet. 47, $305,2015)$. The relevant reasons for citing pertinent publications should also be introduced early in the article rather than discussed as late afterthoughts. This best practice will often entail making statements that are strongly supported by prior publications in the background introducing your findings. We believe this is key to writing research papers with impact that can benefit from peer review, as it encourages explanation of the knowledge gap that motivates the research as well as clear explanation of the conceptual advances made by the main findings of the new research. Similarly, including strong published support for contradictory claims forces proper discussion of the merits of your experimental approaches and the nature of the evidence supporting your own claims. Minimizing or avoiding prior works does no justice to your own achievements and casts doubt on your understanding of your own field of study.

Authors are of course free to select the literature that is relevant to their current work and to cite in their arguments only those publications that meet their standards of evidence and quality. Editors encourage referees to use their experience in the field to ensure that authors are made aware of all relevant prior publications and standards. We do insist as a condition of publication that works brought to our attention be included and accurately represented, but we do not prescribe the mix of self- and other citation nor the journal or preprint archive in which the cited work appears. We maintain that articles that accurately represent the state and main achievements of their field will be the ones trusted, cited and used by other researchers. 